\title{
Article \\ Cytochrome P450s Are Essential for Insecticide Tolerance in the Endoparasitoid Wasp Meteorus pulchricornis (Hymenoptera: Braconidae)
}

\author{
Xiaorong Xing ${ }^{1}$, Mengwen Yan ${ }^{1}$, Huilin Pang ${ }^{1}$, Fu'an Wu ${ }^{1,2}$, Jun Wang ${ }^{1,2}$ (i) and Sheng Sheng $1,2, * \mathbb{C}$ \\ 1 Jiangsu Key Laboratory of Sericultural Biology and Biotechnology, School of Biotechnology, \\ Jiangsu University of Science and Technology, Zhenjiang 212018, China; 172211801110@stu.just.edu.cn (X.X.); \\ 182211802111@stu.just.edu.cn (M.Y.); 182211803105@stu.just.edu.cn (H.P.); 198700001882@just.edu.cn (F.W.); \\ wangjun@just.edu.cn (J.W.) \\ 2 The Key Laboratory of Silkworm and Mulberry Genetic Improvement, Ministry of Agriculture, \\ Sericultural Research Institute, Chinese Academy of Agricultural Science, Zhenjiang 212018, China \\ * Correspondence: shengsheng@just.edu.cn
}

Citation: Xing, X.; Yan, M.; Pang, H.; Wu, F.; Wang, J.; Sheng, S. Cytochrome P450s Are Essential for Insecticide Tolerance in the Endoparasitoid Wasp Meteorus pulchricornis (Hymenoptera: Braconidae). Insects 2021, 12, 651. https://doi.org/10.3390/insects 12070651

Received: 10 June 2021

Accepted: 13 July 2021

Published: 16 July 2021

Publisher's Note: MDPI stays neutral with regard to jurisdictional claims in published maps and institutional affiliations.

Copyright: (c) 2021 by the authors. Licensee MDPI, Basel, Switzerland. This article is an open access article distributed under the terms and conditions of the Creative Commons Attribution (CC BY) license (https:// creativecommons.org/licenses/by/ $4.0 /)$.
Simple Summary: P450s are involved in the detoxification process in insects; however, less attention has been paid for their roles in parasitoid wasps. Here, we identified $28 \mathrm{P} 450$ s from the previous constructed transcriptome dataset of Meteorus pulchricornis and validated their expression patterns under insecticides exposure. Furthermore, we silenced CYP369B3 in adult wasps and demonstrated that the knocking down CYP369B3 significantly increased of the mortality of adult wasps when they were exposed to each chemical insecticide, phoxim, cypermethrin, and chlorfenapyr, respectively. The present study provides a theoretical base for functional research on P450 genes in parasitoid wasps.

Abstract: With the widespread application of insecticides, parasitoid wasps may also be under risk when exposed to insecticides directly at their free-living stages. The endoparasitoid wasp Meteorus pulchricornis is the predominant natural enemy of many lepidopteran pests, such as Spodoptera litura and Helicoverpa armigera. The cytochrome P450 monooxygenases constitute a ubiquitous and complex superfamily of hydrophobic, haem-containing enzymes. P450s are involved in the detoxification of many xenobiotics. However, their exact roles in the tolerance mechanism in parasitoids toward insecticides has received less attention. Here, 28 P450 genes in M. pulchricornis were identified from a previously constructed transcriptome dataset. These P450 genes belonged to CYP2, -3 , and -4 , and mitochondrial clans. Subsequently, eight candidate MpulCYPS were selected from four CYP clans to validate their expression patterns under phoxim, cypermethrin, and chlorfenapyr exposure by qRT-PCR. The results showed that all three insecticides had significant effects on the expression of MpulCYPs. To further study the function of P450s, CYP369B3 was silenced, and its expression levels of CYP369B3 were significantly decreased. Survival analysis indicated that after dsRNA injection, the mortality rate of wasps was significantly increased when M. pulchricornis females were exposed to insecticides compared to control groups. Our findings provide a theoretical base for elucidating the mechanism of insecticide tolerance and promote functional research on P450 genes in parasitoid wasps.

Keywords: Meteorus pulchricornis; cytochrome P450s; detoxification; insecticide tolerance

\section{Introduction}

Mulberry foliage is the exclusive food resource of the silkworm Bombyx mori (Lepidoptera: Bombycidae) and is easily damaged by mulberry pests, resulting in a great threat for the sericulture industry. Among these, the common cutworm Spodoptera litura (Lepidoptera: Noctuidae) has destroyed mulberry much more severely in recent years in China. Therefore, effective control of this pest is vital. Traditional chemical control often 
fails due to the lack of novel effective insecticides and high resistance towards commonly used insecticides in S. litura. Alternatively, other control methods are urgently needed. For decades, biological control agents, such as parasitoid wasps, have been shown to suppress the population of S. litura successfully. In mulberry fields, many parasitoid wasps of $S$. litura have been investigated, and attempts to use them to control $S$. litura have also been promoted.

An effective integrated pest management (IPM) practice is based on balancing the use of chemical insecticides and parasitoid wasps, aiming to create a favorable condition for biological agents. It is well accepted that chemical insecticides usually pose a great challenge for parasitoids not only for survival but also for parasitism factors, such as the parasitism rate or foraging behavior. In other words, releasing parasitoid wasps depends on a clear understanding of their physiology under the pressure of insecticides. Detoxification enzymes are essential for the survival of insects, including parasitoid wasps, when they are exposed to insecticides. In insects, cytochrome P450 monooxygenases (P450s) have received considerable attention for their roles in the detoxification of insecticides, plant secondary metabolites, and toxic pollutants [1]. Cytochrome P450s, also known as mixed function oxidases (MFO), are terminal oxidases in the cytochrome P450 enzyme family. The structure of cytochrome P450s contain nearly $13 \alpha$-helices and $4 \beta$-sheets, among which the highly conserved core region includes haem-binding motifs (FxxGxxxCxG), PERF (PxxFxPE/DRF), Helix-I (A/GGxD/ETT/S), Helix-K (ExxR), and Helix-C (WxxxR) [2]. Based on their phylogenetic classification, insect $\mathrm{P} 450 \mathrm{~s}$ can be divided into four major clans: CYP2, CYP3, CYP4, and mitochondrial clans. In insects, P450s are also involved in the biosynthetic and catabolic processes of juvenile hormones, ecdysterone, and cuticular hydrocarbon, which regulate insect growth and development [3].

Determining the gene expression level under insecticide stress is a common method to evaluate whether detoxification enzymes are involved in insect resistance [4,5]. Exposure to an $\mathrm{LD}_{5}$ dose of lambda-cyhalothrin significantly upregulated five P450 genes (CYP4M59, CYP6AE119, CYP6AE120, CYP6AE121, and CYP6BD18) in Pieris rapae larvae [6]. $\lambda$-Cyhalothrin exposure enhanced $P 450$ activity and induced the expression of $C Y P 6 A B 12$ significantly in S. litura larvae [7]. The expression level of P450s in Bemisia tabaci with resistance to neonicotinoid insecticides was higher than that of sensitive strains [8]. However, compared to insect pests, fewer studies have been conducted to elucidate the sensitivity and detoxification mechanisms of parasitoid wasps toward insecticides.

In mulberry fields, phoxim (benzoyl cyanide-O-(diethoxyphosphinothioyl)oxime), cypermethrin (cyano-3-phenoxybenzyl-2,2-dimethyl-3-(2,2-dichlorovinyl)cyclopropanecar boxylate), and chlorfenapyr (4-bromo-2-(4-chlorophenyl)-1-ethoxymethyl-5-trifluoromethy lpyrrole-3-carbonitrile) are commonly used insecticides in chemical pest control [9]. The solitary endoparasitoid M. pulchricornis (Hymenoptera: Braconidae) is the predominant natural enemy of many lepidopteran pests, such as S. litura, Helicoverpa armigera, and Lymantria dispar. It has been reported that S. litura can be attacked by M. pulchricornis at a rate of 20-30\% in soybean fields [10]. Previous studies demonstrated that glutathione s-transferase genes were upregulated after $M$. pulchricornis females were exposed to commonly used insecticides in mulberry fields, such as phoxim and cypermethrin [11]. However, the roles of P450s in M. pulchricornis when they are under insecticide stress remain unknown.

The present study aimed to identify the candidate P450 genes in M. pulchricornis and validate their expression levels when female wasps were treated with commonly used insecticides in mulberry. Furthermore, RNA interference was performed to investigate the function of P450s by silencing the expression of selected P450s genes. The information gained from this study can improve the understanding of the detoxification mechanism of M. pulchricornis and provide a reference for the protection of parasitoid wasps. 


\section{Materials and Methods}

\subsection{Insects}

Since M. pulchricornis is thelytokous, all stocks of this parasitoid are female [12,13]. Parasitoid adults were reared on the larvae of $S$. litura, which were collected from a mulberry field in Jiangsu University of Science and Technology (Zhenjiang, China). The adult parasitoid wasps were fed a $10 \%(w / w)$ honey solution as a supplement in glass culture tubes (2.2-cm diameter, $8-\mathrm{cm}$ height, plugged with gauze). S. litura larvae were fed an artificial diet in plastic cases (13-cm length, 13-cm width, and 6-cm height) [14], and the adults were furnished with strips of paper as a substrate for oviposition in organza-covered cages with a $10 \%$ honey solution $(20-\mathrm{cm}$ length, $20-\mathrm{cm}$ width, and $30-\mathrm{cm}$ height) [10]. Round sheets of mulberry leaves $(6.5-\mathrm{cm}$ diameter) were placed in plastic boxes $(6.5-\mathrm{cm}$ diameter) with punched lids and lined with agar gel (3\%). Each box contained twenty third-instar S. litura larvae and two fifteen-day-old wasps for parasitism. Insects were reared after $8 \mathrm{~h}$ of parasitism. The rearing conditions of both insects were $25 \pm 1{ }^{\circ} \mathrm{C}, 60-80 \%$ $\mathrm{RH}$, and a photoperiod of 14:10 h (L:D).

\subsection{Identification of $P 450$ Genes in M. pulchricornis}

P450 genes were identified from the previously constructed transcriptome database of M. pulchricornis (GenBank accession number: SRR8981255; [11]). The tBLASTn algorithm (E value $<10^{-5}$ ) was used to identify candidate unigenes encoding putative P450s in M. pulchricornis against the reference sequences of Apis mellifera, Drosophila melanogaster, Nasonia vitripennis, and $S$. litura. Putative genes were checked manually using the BlastX program by the nr database to confirm their identity with other insect P450 genes. According to the conservative motif of insects, the genes with the characteristic signal sequence of insect P450s were identified as the candidate P450 genes in M. pulchricorni. All identified P450 genes were named and classified by the Cytochrome P450 Nomenclature Committee.

\subsection{Sequence and Phylogenetic Analyses}

The ORF Finder (https:/ / www.ncbi.nlm.nih.gov / orffinder/, accessed on 18 October 2020) was used to predict the open reading frames (ORFs) of putative $P 450$ genes, and ExPASy (www.expasy.org/tools/protparam.html, accessed on 18 October 2020) was used to predict the molecular weight and theoretical isoelectric point (pI). SignalP (https: / / www. ncbi.nlm.nih.gov/Structure/bwrpsb/bwrpsb.cgi, accessed on 18 October 2020) was used to predict the N-terminal signal peptide and the CD-Search tool (https:/ / www.ncbi.nlm.nih. gov/Structure/bwrpsb/bwrpsb.cgi, accessed on 18 October 2020) to predict conservative domains. A phylogenetic tree was constructed by MEGA7.0 software using the neighborjoining method with 1000 bootstrap replications [15] and decorated by FigTree1.43.

\subsection{Insecticide Treatment}

Phoxim (purity, 99\%) and cypermethrin (purity, 99.7\%) analytical standards were purchased from Aladdin (Shanghai, China). Chlorfenapyr (purity, 99\%) was obtained from Nanjing Agricultural University, China. According to our previous results, the $\mathrm{LC}_{10}$ of phoxim and cypermethrin was 0.1 and $2.0 \mathrm{mg} / \mathrm{L}$, respectively [16], and these two chemicals were dissolved in analytical-grade acetone to prepare working solution. Due to chlorfenapyr being newly promoted in the mulberry fields for controlling the pests, no sublethal dose under indoor condition can be acquired. Therefore, the appropriately $10 \%$ field application dose $(4 \mathrm{mg} / \mathrm{L})$ [17] was prepared to obtain the chlorfenapyr working solution which was also dissolved in acetone. The tested wasps were originally kept in glass tubes (2.2-cm diameter, $8-\mathrm{cm}$ height, plugged with gauze) and the tubes were transferred to the ice surface. After 20-30 s, these wasps became inactive and then they were taken out from tubes to be conducted for the further tests. Next, 1- $\mu \mathrm{L}$ insecticide solutions were topically applied to the tergum using a microsyringe (Sangon Biotech, Shanghai, China). Wasps treated with $1 \mu \mathrm{L}$ of acetone were used as controls. Post-treatment, all live parasitoid wasps were reared separately in the previously described above conditions and fed a $10 \%$ 
$(w / w)$ honey solution. The survivors were collected for further analyses. Ten female adults constituted one biological sample and three replicates were tested.

\section{5. qRT-PCR Validation}

Total RNA was extracted using RNAiso Plus reagent (Takara Biotechnology Co. Ltd., Dalian, China), in accordance with the manufacturer's protocol, from the whole bodies of surviving wasps after the 24-h insecticide exposure above mentioned. Approximately five survivors were pooled to prepare one sample. RNA quality and concentration were determined using a Nanodrop 2000 spectrometer (Thermo Fisher Scientific, New York, NY, USA). Each RNA sample was reverse transcribed using a PrimeScript RT reagent kit with gDNA Eraser (Takara, China) and then diluted to $70 \mathrm{ng} / \mathrm{L}$ with RNase-free $\mathrm{H}_{2} \mathrm{O}$. qRT-PCR was performed on a QuantStudio ${ }^{\mathrm{TM}}$ Real-Time PCR system (Applied Biosystems, Foster City, CA, USA) using SYBR Premix Ex Taq II (Tli RNaseH Plus; Takara Biotechnology Co. Ltd., Dalian, China), in accordance with the manufacturer's instructions. Each reaction ( $20 \mu \mathrm{L}$ volume) was constituted by $10 \mu \mathrm{L}$ of SYBR Premix Ex Taq II, $0.4 \mu \mathrm{L}(10 \mu \mathrm{M})$ of each primer (Supplementary Table S1), $1.5 \mu \mathrm{L}(75 \mathrm{ng})$ of cDNA template, $0.4 \mu \mathrm{L}(50 \times)$ of ROX reference dye, and $7.3 \mu \mathrm{L}$ of $\mathrm{RNase}-$ free $\mathrm{H}_{2} \mathrm{O}$. The reaction program parameters were as follows: $95^{\circ} \mathrm{C}$ for $5 \mathrm{~min}$ and 45 cycles of $95^{\circ} \mathrm{C}$ for $15 \mathrm{~s}$ and $60^{\circ} \mathrm{C}$ for $31 \mathrm{~s}$. A notemplate control and a no-reverse transcriptase control were both included on each reaction plate to test the underlying contamination. Reactions for all samples were independently repeated three times. M. pulchricornis $\beta$-actin (Acc: AQN78522.1) was chosen as the internal reference gene to normalize the target gene expression. The relative expression levels of MpulCYP genes among the different samples were measured using the $2^{-\Delta \Delta C t}$ method [18]. Differences in the expression level of each target gene between the treatments were compared by one-way analysis of variance (Systat, Inc., Evanston, IL, USA) with Tukey's post hoc test $(p<0.05)$ using R version 3.4.0 [19]. All the data were tested for the normality and homogeneity of variance and log or square root transformations were necessary to satisfy the hypothesis of normality and homogeneity of variance.

\section{6. dsRNA Synthesis and Injection RNA Interference}

RNA interference (RNAi) was conducted to further analyze the functions of P450s in M. pulchricornis. Gene-specific primers for dsRNA synthesis were designed with BLOCKiT $^{\mathrm{TM}}$ RNAi Designer (https://rnaidesigner.thermofisher.com/, accessed on 10 January 2021). The single-stranded Oligos of genes were synthesized following the manufacturer's instructions and are listed in Supplementary Table S2. dsRNA was synthesized using an In Vitro Transcription T7 Kit (for siRNA synthesis) (Takara Biotechnology Co. Ltd., Dalian, China), and the concentration purity of dsRNA was assessed by a NanoDrop 2000 spectrophotometer. The dsRNA quality was further examined by $2 \%$ agarose gel electrophoresis, and the dsRNA was subsequently stored at $-20{ }^{\circ} \mathrm{C}$ until use. Before dsRNA injection, tested wasps were kept in the glass tubes and these tubes were transferred to the ice surface for 20-30 s as mentioned above and then dsRNA was injected. Next, $0.5 \mu \mathrm{L}$ dsRNA ( $50 \mathrm{ng}$ /individual) was injected into the 2-day-old adults using a Nanoject II (Drummond Scientific, Broomall, PA, USA) with a microglass needle (0.53-mm inner diameter, 1.14-mm external diameter). After dsRNA injection, these wasps were collected and reared in a glass tube $(2.2-\mathrm{cm}$ diameter, $8-\mathrm{cm}$ height, plugged with gauze) separately by feeding a $10 \%(w / w)$ honey solution. Total RNA was extracted after 12,24 , and $48 \mathrm{~h}$ to check the efficiency of RNAi by qRT-PCR. The dsRNA of the green fluorescent protein gene (GFP) was also synthesized as a negative control. M. pulchricornis adults were individually treated with $1 \mu \mathrm{L}$ of the insecticide solution after $12 \mathrm{~h}$ of injection, and the method was the same as above. The death of individual wasps was recorded daily, and mortality rates were calculated as the mortality after $24 \mathrm{~h}$ of treatment. Each replicate included 10 wasps and was replicated three times. 


\section{Results}

\subsection{Identification of P450s and Phylogenetic Analyses}

A total of 28 P450 genes in M. pulchricornis (Table 1) were identified. Sequences of CYPs identified here were deposited in GenBank, and the accession numbers are listed in Table 1. All 28 genes contained full-length ORFs. The length of deduced MpulCYP proteins ranged from 209 to 557 amino acid residues. The theoretical pI ranged from 5.77 to 9.31 , and the predicted molecular weight ranged from 23.92 to $116.83 \mathrm{kD}$. The BLASTx results showed that there were 22 P450 genes of M. pulchricornis which shared high sequence identity with P450s in $M$. demolitor. The results of conserved domain analyses revealed that 26 P450 genes had haem-binding motifs, 27 had PERF motifs, 21 had helix-C motifs, and all genes had helix-K and helix-I motifs (Figure 1).

\begin{tabular}{|c|c|c|c|c|c|c|}
\hline & Name & Helix C & Helix I & Helix K & PERF Site & Heambinding Domair \\
\hline & & 125 & 450 & 510 & 560 & 595 \\
\hline ح & MpulCYP305D1 & WREMR & GLQTT & ESQR & PQSFKPERH & FFGGGHRRCPG \\
\hline & MpulCYP369B3 & WQEQR & ALSAV & ETLR & PDIFRPERF & PFGFGRRVCAG \\
\hline & MpulCYP6AS186 & WGPLR & GEETS & ETLR & PDVFDPERF & SFGQGPRNCIG \\
\hline & MpulCYP6AS187 & WQPLR & SEATS & ETLR & PDKFDPERF & AFGAGPRNCIG \\
\hline & MpulCYP6AS188 & WRLLR & GEETS & ETLR & PDVFDPERF & SFGDGPRNCIG \\
\hline & MpulCYP6AS189 & WRPLR & GEETS & ETLR & PDKFDPARF & SFGSGPRNCIG \\
\hline & MpulCYP6AS190 & WRPLR & GEETS & ETLR & PDVFDPERF & SFGEGPRNCIG \\
\hline & MpulCYP6BC12 & WKNLR & GYQPS & ETLR & PDKFNPDRF & PFGEGPRNCIG \\
\hline & MpulCYP6JT7 & WKVLR & GEETA & ETLR & PDVFDPERF & PFGHGPKNCIG \\
\hline & MpulCYP6SP5 & WKYLR & GLESS & ETMR & PEKFDPDRF & PFGEGPRICIG \\
\hline & MpulCYP6SP6 & WKYLR & GLESS & ETLR & PEKFDPDRF & PFGEGPRICIG \\
\hline & MpulCYP 6SQ-1 & ----- & GFESS & EGVR & PDVFNPD-- & PFGIGPRMCVG \\
\hline & MpulCYP9P37 & WREVR & GEDTS & ETLR & PDKFDPERF & PFGVGPRKCIG \\
\hline & MpulCYP9R94 & WREIR & GEDTS & ETLR & PKKFSPERF & PFGDGPRICIG \\
\hline & MpulCYP9R95 & WRETR & GFEST & ETLR & PDEFDPERF & PFGLGPRMCIG \\
\hline & MpulCYP336X1 & WKNER & GYLTS & ESMR & PEKFLPERF & PFGGGARICPG \\
\hline & MpulCYP4G283 & WRAHR & GHDTT & ETLR & PDTFNPDNF & PFSAGPRSCVG \\
\hline & MpulCYP4G284 & WRYHR & GHDTT & ESLR & PEVFDPDRF & PFSAGPRSCVG \\
\hline & MpulCYP4AV17 & ----- & GHDTV & ETWR & PESFLPERF & PFSAGSRNCIG \\
\hline U & MpulCYP4FQ8 & WRAGR & GHDTT & ESLR & PNVYDPDRF & PFSGGPRNCIG \\
\hline & MpulCYP4249-1 & $----\overline{-}$ & FYDAV & EALR & PDKFIPERF & PFSGGSRTCPA \\
\hline & MpulCYP4249-2 & ----- & YGDTI & ETIR & PDKFIPERF & PFSGGSRSCAG \\
\hline & MpulCYP4249-3 & MKARR & FHDTI & ETMR & --------- & ---------- \\
\hline & MpulCYP12-1 & $----\overline{-}$ & GIDTT & ESLR & SRA------- & -ーーーーーーーーー \\
\hline & MpulCYP301A1 & WKEER & GIDTI & EVER & AGSFTPERW & PYGYGARMCLG \\
\hline & MpulCYP301B1 & WSEER & GIDTT & ETER & CNEFLPERW & PFGFGRRMCLG \\
\hline & MpulCYP302A1 & ----- & GIDTT & ESER & PNCF I PERW & PFGHGPRSCIA \\
\hline & MpulCYP315A1 & ----- & AGDTT & ESLR & PDNFLPERW & PFAMGVRSCIG \\
\hline
\end{tabular}

Figure 1. Alignment of functional domains of the $28 \mathrm{MpulCYPs}$. Conserved motifs are indicated in shadows.

A neighbor-joining tree was constructed using the protein sequences of $28 \mathrm{MpulCYPs}$ and P450s from four other insect species, namely S. litura, A. mellifera, D. melanogaster, and N. vitripennis (Figure 2). Of these, the CYP2, CYP3, CYP4, and mito clans contained 2, 13, 7 , and 6 genes, respectively. The CYP2 and mito clans had fewer P450 genes compared with the CYP3 and CYP4 clans. There were two clusters, CYP305 and CYP369, in the CYP2 clan. In the CYP3 clan, the two largest clusters were CYP6 and CYP9. In the CYP4 clan, most genes were from the CYP4 cluster, and in the mito clan, the clusters included CYP12, CYP301, CYP302, and CYP315. Phylogenetic analysis revealed a close relationship between P450 genes from M. pulchricornis and N. vitripennis. Some P450 genes were orthologs, including MpulCYP6AS187 and NvitCYP6AS34 in the CYP3 clan, MpulCYP4FQ8 and NvitCYP4AB7 in the CYP4 clan, and MpulCYP301B1 and NvitCYP301B1 in the mito clan. 
Table 1. Sequence information of identified MpulCYP genes.

\begin{tabular}{|c|c|c|c|c|c|c|c|c|c|}
\hline \multirow[b]{2}{*}{ Gene Name } & \multirow{2}{*}{ Acc. No } & \multirow{2}{*}{$\begin{array}{l}\text { Encoded Protein } \\
\text { Length/aa }\end{array}$} & \multirow{2}{*}{$\begin{array}{l}\text { Molecular } \\
\text { Weight/kD }\end{array}$} & \multirow[b]{2}{*}{ Isoelectric Point } & \multicolumn{5}{|c|}{ Blastx Best Hit } \\
\hline & & & & & Species & Protein Name & E Value & Identity \% & Accession \\
\hline MpulCYP4G283 & MZ169337 & 557 & 63.23 & 7.65 & Fopius arisanus & cytochrome P450 4g15 & 0 & 89.61 & XP_011304723.1 \\
\hline MpulCYP4G 284 & MZ169336 & 555 & 63.44 & 6.57 & Microplitis demolitor & cytochrome P450 4g15 & 0 & 80.29 & XP_014300333.1 \\
\hline MpulCYP4AV17 & MZ169338 & 313 & 35.86 & 6 & Microplitis demolitor & cytochrome P450 4c3-like & $1.00 \times 10^{-155}$ & 69.01 & XP_008555432.1 \\
\hline MpulCYP6AS186 & MZ169329 & 504 & 58.35 & 9.18 & Microplitis demolitor & probable cytochrome P450 6a14 & 0 & 59.84 & XP_008560120.1 \\
\hline MpulC YP6AS187 & MZ169330 & 500 & 57.2 & 9.25 & Microplitis demolitor & probable cytochrome P450 6a14 & 0 & 56.10 & XP_008560120.1 \\
\hline MpulCYP6AS188 & MZ169333 & 500 & 57.59 & 9.15 & Microplitis demolitor & cytochrome P450 6k1 & 0 & 57.75 & XP_008557726.1 \\
\hline MpulCYP6AS189 & MZ169331 & 499 & 57.42 & 8.28 & Microplitis demolitor & probable cytochrome P450 6a14 & 0 & 67.34 & $\mathrm{XP}_{-}^{-} 008560120.1$ \\
\hline MpulCYP6AS190 & MZ169332 & 502 & 58.13 & 8.49 & Microplitis demolitor & probable cytochrome P450 6a14 & 0 & 68.76 & XP_008560120.1 \\
\hline MpulC YP6BC12 & MZ169334 & 522 & 60.41 & 8.85 & Polistes dominula & cytochrome P450 6B5-like & 0 & 65.31 & XP_015174482.1 \\
\hline MpulCYP6SP5 & MZ169326 & 512 & 58.99 & 9.18 & Diachasma alloeum & cytochrome P450 6k1 & 0 & 61.05 & XP_015121128.1 \\
\hline MpulCYP6SP6 & MZ169327 & 502 & 58.3 & 9.31 & Diachasma alloeum & cytochrome P450 6k1 & 0 & 57.49 & XP_015121128.1 \\
\hline MpulCYP6SQ-1 & MZ169325 & 268 & 30.38 & 6.13 & Orussus abietinus & cytochrome P450 6k1-like & $3.00 \times 10^{-139}$ & 70.63 & XP_012272474.2 \\
\hline MpulС YР9РЗ & MZ169322 & 505 & 58.95 & 7.58 & Belonocnema treatae & cytochrome P450 9e2-like & 0 & 58.16 & XP_033219303.1 \\
\hline MpulC YP9R94 & MZ169321 & 508 & 58.7 & 7.97 & Microplitis demolitor & cytochrome P450 9e2-like & 0 & 61.55 & XP_008557951.2 \\
\hline MpulCYP9R95 & MZ169323 & 507 & 58.28 & 6.9 & Microplitis demolitor & cytochrome P450 9e2-like & 0 & 58.65 & XP_o08557951.2 \\
\hline MpulCYP12-1 & MZ169346 & 224 & 25.57 & 9.16 & Microplitis demolitor & cytochrome P450 CYP12A2 & $8.00 \times 10^{-96}$ & 71.14 & XP_008548500.1 \\
\hline MpulCYР301A1 & MZ169345 & 525 & 60.42 & 8.48 & Microplitis demolitor & $\begin{array}{l}\text { probable cytochrome P450 301a1, } \\
\text { mitochondrial }\end{array}$ & 0 & 83.69 & XP_008548437.1 \\
\hline MpulCYР301B1 & MZ169342 & 513 & 60 & 8.8 & Microplitis demolitor & probable cytochrome P450 49a1 & 0 & 76.26 & XP_014298339.1 \\
\hline МриІСҮРЗО2А1 & MZ169344 & 302 & 34.26 & 8.19 & Microplitis demolitor & cytochrome P450 302a1, mitochondrial & $1.00 \times 10^{-141}$ & 66.01 & XP_008552208.1 \\
\hline MpulCYР336X1 & MZ169324 & 504 & 58.01 & 8.55 & Microplitis demolitor & cytochrome P450 9e2 & 0 & 0.5029 & XP_008557820.1 \\
\hline МриіСҮРЗб9В3 & MZ169320 & 505 & 116.83 & 6.75 & Microplitis demolitor & probable cytochrome P450 304a1 & 0 & 59.12 & XP_008561134.1 \\
\hline MpulCYP4249-1 & MZ169335 & 258 & 29.53 & 8.64 & Microplitis demolitor & cytochrome P450 4C1-like & $9.00 \times 10^{-105}$ & 57.60 & XP_014298773.1 \\
\hline MpulCYP4249-2 & MZ169340 & 209 & 23.92 & 8.91 & Microplitis demolitor & cytochrome P450 4C1-like & $6.00 \times 10^{-73}$ & 52.48 & XP_014298773.1 \\
\hline MpulCYP4249-3 & MZ169341 & 301 & 34.15 & 5.77 & Microplitis demolitor & cytochrome P450 4C1-like & $4.00 \times 10^{-92}$ & 48.44 & XP_014298773.1 \\
\hline
\end{tabular}




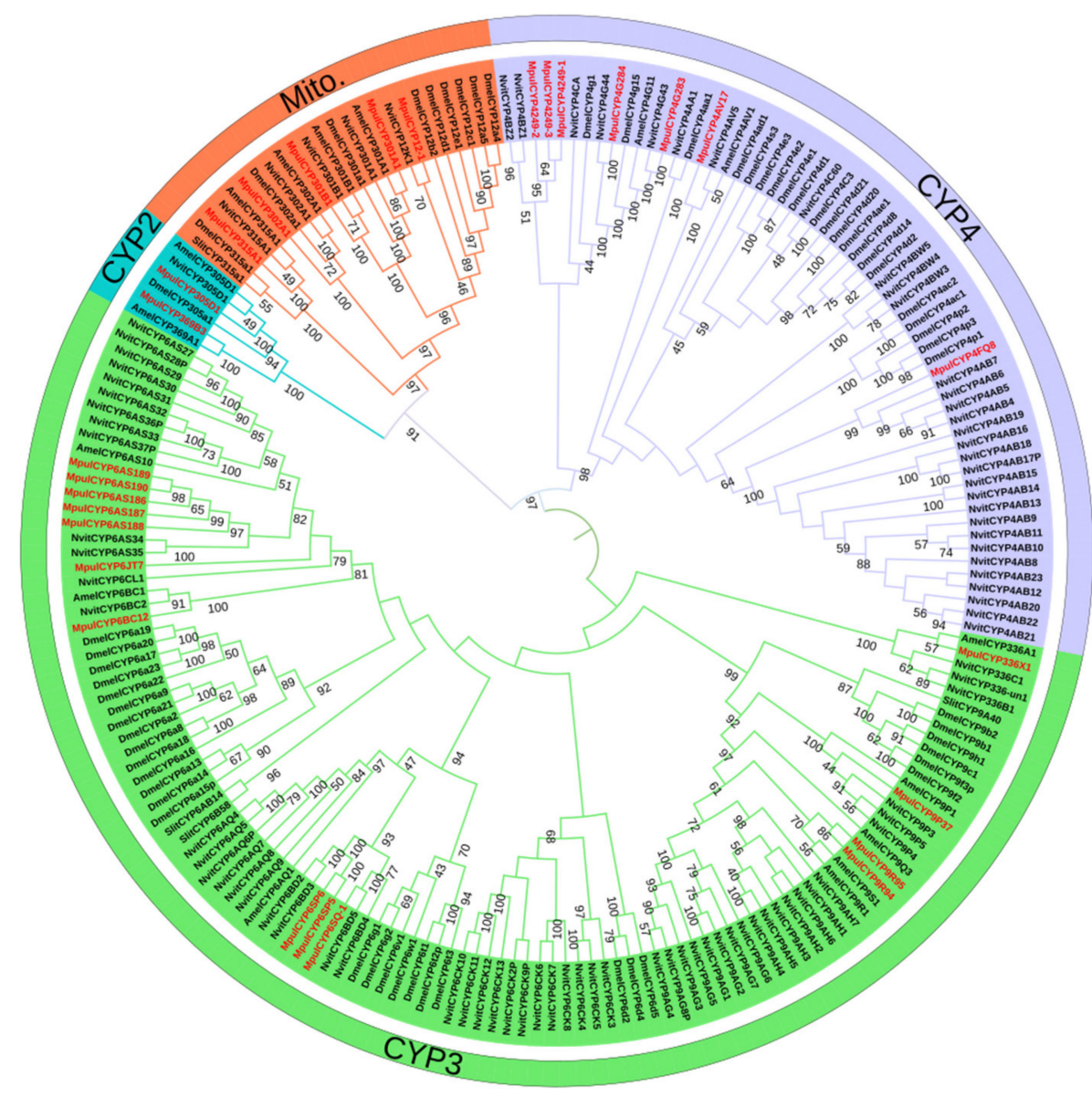

Figure 2. Phylogenetic analysis of P450s. Meteorus pulchricornis (Mpul), Spodoptera litura (Slit), Apis mellifera (Amel), Drosophila melanogaster (Dmel), Nasonia vitripennis (Nvit). Different colors indicate CYPs in different clans.

3.2. Relative Expression Profiling of P450s after Phoxim, Cypermethrin and Chlorfenapyr Treatment

Three insecticides, namely phoxim, cypermethrin, and chlorfenapyr, were chosen to quantitatively measure the expression levels of the eight candidate MpulCYPs from four CYP clans by qRT-PCR (Figure 3). In response to phoxim exposure, the expression levels of seven MpulCYPs (MpulCYP305D1, MpulCYP369B3, MpulCYP4249-1, MpulCYP6SP6, MpulCYP302A1, CYP9R94, and MpulCYP315A1) were upregulated by 1.39-4.72-fold compared to the acetone control. The expression levels of MpulCYP4FQ8 were downregulated by $66 \%$. In response to cypermethrin exposure, the expression levels of six MpulCYPS (MpulCYP305D1, MpulCYP369B3, MpulCYP4249-1, MpulCYP6SP6, MpulCYP302A1, and CYP9R94) were upregulated by 1.4-118.89-fold compared to the acetone control. Remarkably, the expression levels of MpulCYP369B3 were upregulated by 118.89 -fold. The expression levels of MpulCYP4FQ8 and MpulCYP315A1 were reduced by 31\% and 35\%, respectively. In response to chlorfenapyr exposure, the expression levels of six MpulCYPS (MpulCYP369B3, MpulCYP4FQ8, MpulCYP4249-1, MpulCYP6SP6, MpulCYP302A1, and CYP9R94) were upregulated by 1.13-486.91-fold compared to the control. The expression levels of two MpulCYPs (MpulCYP305D1 and MpulCYP315A1) were reduced by 36\% and $42 \%$, respectively. The expression levels of MpulCYP4FQ8 were upregulated 486.91fold. It is noted that MpulCYP369B3, MpulCYP4249-1, MpulCYP6SP6, MpulCYP302A1, and CYP9R94 were upregulated by phoxim, cypermethrin, and chlorfenapyr exposure simultaneously. 
Insects 2021, 12, 651

8 of 13
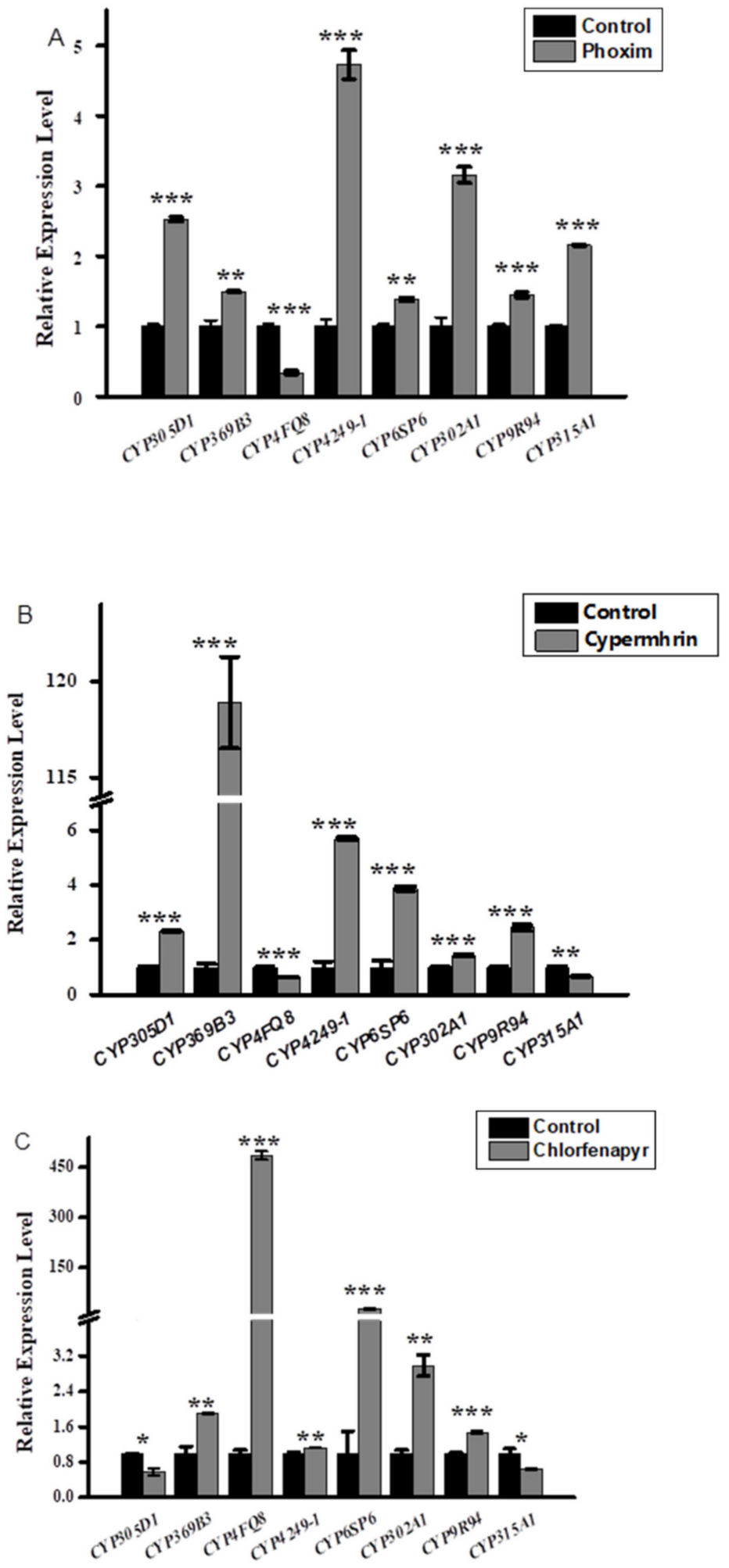

Figure 3. Relative expression profiles of MpulCYPs after phoxim (A), cypermethrin (B), and chlorfenapyr (C) treatment. The transcriptional level of each gene in insecticide-treated individuals was normalized relative to that in acetone-treated (control) individuals. Asterisks indicates a significant difference in relative expression levels (one-way analysis of variance with Tukey's post hoc test, $\left.{ }^{*} p<0.05 ;{ }^{* *} p<0.01 ;{ }^{* * *} p<0.001\right)$. Log transformation was applied to satisfy the hypothesis of normality. 


\subsection{RNAi for CYP369B3}

To investigate the roles of MpulCYPs in insecticides detoxification, CYP369B3 was selected and successfully silenced by RNAi in 2-day-old adult wasps. The results showed that RNAi treatment significantly decreased the expression levels of CYP369B3 $12 \mathrm{~h}$ after dsRNA injection. Although the expression levels of CYP369B3 were decreased 24 and $48 \mathrm{~h}$ after silencing, there were no significant differences between dsRNA and dsGFP injection groups (Figure 4). Furthermore, after $d_{s} C Y P 369 B 3$ injection, the mortality adult was increased by $114.27 \%, 74.39 \%$, and $300.30 \%$ compared to $d s G F P$ injection groups when they exposed to phoxim, cypermethrin, and chlorfenapyr, respectively (Figure 5).

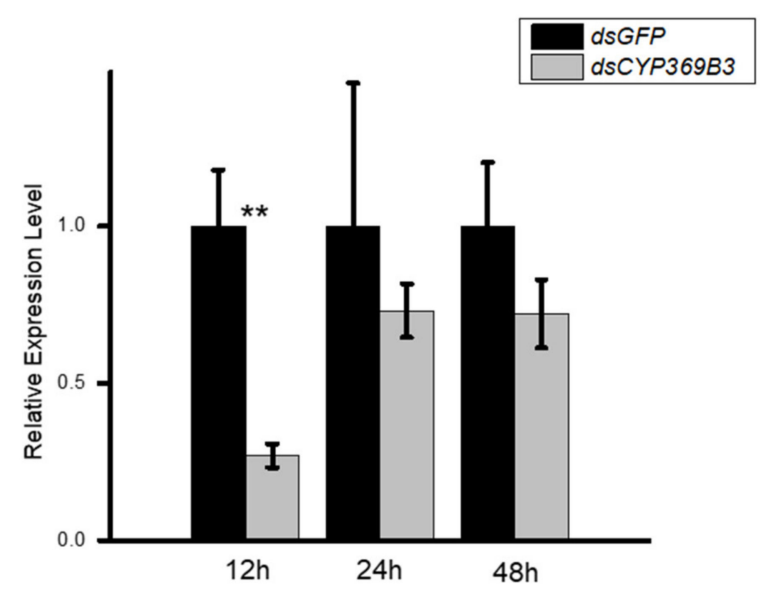

Figure 4. The analysis of the expression of CYP369B3 after RNAi. Differences in the expression levels of each target were compared using a $t$-test. Significant differences are indicated by asterisks $(* * p<0.01)$.

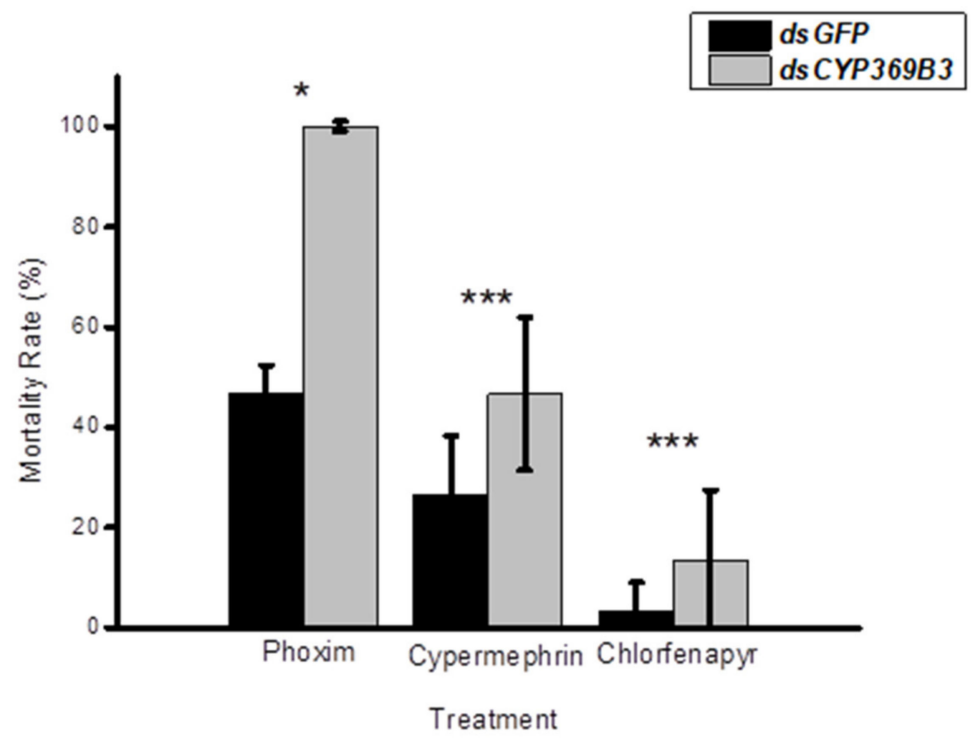

Figure 5. Effects of knocking down CYP369B3 on the mortality of Meteorus pulchricornis adults after treatment with insecticides. ${ }^{*} p<0.05 ;{ }^{* * *} p<0.001$ ). Square root transformation was applied to satisfy the hypothesis of normality.

\section{Discussion}

The extensive application of insecticides caused significant negative effects on the natural enemies of pests [20]. Thus far, many insecticides have been found to have strong toxicity to parasitic wasps. For instance, phoxim and cypermethrin were reported to be 
harmful to M. pulchricornis [16]. Yang et al. [21] demonstrated that chlorpyrifos, chlorantraniliprole, emamectin benzoate, and spinosad were toxic to Trichogramma japonicum and Trichogramma dendrolimi. Although the lethal and/or sublethal effects of insecticides on parasitoid wasps have been tested in a wide range of insecticides, the detoxification mechanisms of parasitoid wasps have received less attention.

P450s have been previously identified in many kinds of insects, and these CYPs were suggested to participate in various physiological processes, such as detoxication [22], pesticide resistance [23,24], immune response [25], and cuticularization [26] in insects. To date, studies regarding the detoxication functions of P450s have mainly focused on agricultural or forestry pests, but there is very limited research on non-target insects, especially on parasitoid wasps.

Identification of P450 genes is a key step in investigating their roles in insects. In this study, 28 CYPs were identified from the transcriptome of M. pulchricornis. The number of P450 genes found in M. pulchricornis differed significantly from the number of CYP genes found in other wasps, such as A. mellifera (50 CYPs; [27]), N. vitripennis (92 CYPs; [28]), Macrocentrus cingulum (41 CYPs), Microplitis demolitor (47 CYPs), and Ceratosolen solmsi (39 CYPs; [29]. This is mainly due to the diverse functions of P450s among insect species [30]. Furthermore, the depth of sequencing may also affect the number of P450s screened here.

It is well known that MpulCYPs are important players in the regulation of the degradation of insecticides in insects. CYP2 clans of P450s are often involved in environmental response, and mito clans are involved in the synthesis of ecdysone [31,32]. In contrast, CYP3 and CYP4 are well known for their multiple roles in ecological adaptation in insects, such as the metabolism of exogenous toxic chemicals from the environment or hosts, participation in fatty acid hydroxylation, and biosynthesis [33]. Therefore, the number of $C Y P 3$ and CYP4 clans identified from M. pulchricornis was dramatically higher than that of the Mito and CYP2 clans.

Phylogenetic analysis revealed a close relationship between P450 genes from M. pulchricornis, A. mellifera, D. melanogaster, and N. vitripennis. The homology between AmelCYP4G11 and MpulCYP4G283 was 100\%, and AmelCYP4G11 may play an important role in the hygiene behavior and antioxidant process of A. mellifera [34]. Therefore, it was speculated that MpulCYP4G283 may play the same role in M. pulchricornis. The homology of MpulCYP6SP6, MpulCYP6SP6, and MpulCYP6SQ-1 was 100\%, and the homology of these three genes to DmelCYP6G1 was 94\%. Overexpression of DmelCYP6G1 in D. melanogaster contributed to the metabolic resistance to dichlorodiphenyltrichloroethane, imidacloprid, acetamiprid, nitenpyram, and malathion [35]. Therefore, it was speculated that these three genes played an important role in insecticide metabolism.

Cytochrome P450 has both a highly conserved core region (haem-binding site and oxygen-binding site) and a variable region associated with substrate recognition and substrate binding (substrate-binding site). The conserved cysteine (Cys) in the haem-binding motif ( $F x x G x x x C x G)$ is responsible for the absorption peak of the CO-binding protein at 450 $\mathrm{nm}$. Helix-I is presumed to be the binding site of molecular oxygen, and helix-K may play a role in stabilizing the core structure of cytochrome P450 proteins [2]. In this study, 21 CYP protein sequences had conservative CYP domains. Studying the structure of different cytochrome P450 monooxygenases not only contributes to the further understanding of the relationship between the structure and function of $\mathrm{P} 450$, but also provides theoretical reference for the development of new insecticides based on the molecular structure of P450.

Studies have shown that the P450 oxidase system is one of the main functional enzymes in insecticide metabolism, which can improve the ability to degrade insecticides by increasing the P450 expression level. In the present study, to further investigate the potential role of P450s in the tolerance mechanism in M. pulchricornis when they suffered from commonly used insecticides in mulberry fields, the expression of P450s in M. pulchricornis was validated after phoxim, cypermethrin, and chlorfenapyr exposure. The results showed that most MpulCYPs significantly increased their expression levels after insecticide stress. In Musca domestica, CYP6D1 was overexpressed in resistant strains of pyrethroid 
and organophosphorus and had a metabolic effect on insecticides [36]. Both CYP6G1 in D. melanogaster and CYP6AY1 in Nilaparvata lugens was upregulated and contributed to the metabolic effect on imidacloprid insecticides [37,38]. Regulating the expression of the P450 gene caused T. vaporariorum to develop imidacloprid resistance. Compared with susceptible strains, CYP6ER1 in N. lugens was significantly overexpressed in the field-collected resistant strains, which further provided a basis for $\mathrm{P} 450$ to participate in the generation of insect resistance [39]. This further provides a strong basis for demonstrating that P450 is involved in the development of insect resistance.

In addition, P450 genes were not only extensively affected by insecticide stress, but also had the function of responding to multiple selection pressures from insecticides. Although the substrates of different P450 genes are different, they may overlap to adapt to environmental changes. The expression of several $\mathrm{P} 450$ genes in response to the induction of exogenous compounds, such as insecticides, may also be used to respond to the selection pressure of insecticides, showing evolutionary plasticity [40]. The expression of 17 P450 genes was significantly upregulated by chlorantraniliprole and fipronil stress in Mythimna separata [41]. Similarly, in the present study, almost all the selected P450s genes in $M$. pulchricornis were upregulated and the expression levels of MpulCYP369B3, MpulCYP42491, MpulCYP6SP6, MpulCYP302A1, and CYP9R94 were significantly increased after exposure to phoxim, cypermethrin, and chlorfenapyr. Therefore, it can be speculated that these P450s genes were involved in the detoxification of phoxim, cypermethrin, and chlorfenapyr.

RNAi technology has been widely used to uncover the functions of genes involved in insect detoxification. Sun et al. [42] evaluated the role of CYP6FU1, CYP425A1, and CYP6AY1 in the resistance of $N$. lugens to etofenprox using RNAi and they found that knocking down these genes resulted in the higher sensitivity to etofenprox. Silencing of the CYP6BG1 gene in Plutella xylostella demonstrated its role in chlorantraniliprole resistance [43]. Using RNAi, Mao et al. [44] evaluated the impacts of CYP6ER1, CYP302A1, and CYP3115A1 in nitenpyram resistance in N. lugens. When the three P450 genes were successfully silenced, the sensitivity of $N$. lugens to nitenpyram was increased. Similarly, the CYP6ER1 gene was demonstrated to involve in the resistance of $N$. lugens to thiamethoxam and dinotefuran, similar to imidacloprid, and the knockdown of CYP6ER1 dramatically increased the toxicity of clothianidin to N. lugens [45]. In this study, MpulCYP369B3 was significantly upregulated by the three insecticides, and expression was upregulated 118-fold after cypermethrin stress. When MpulCYP369B3 was successfully knocked down, the mortality of M. pulchricornis significantly increased. Therefore, MpulCYP369B3 may play an important role in response to insecticide stress in M. pulchricornis.

In summary, the present study reported the large-scale identification of P450 genes in M. pulchricornis, and their expression patterns were significantly affected by insecticide treatment. Knocking down CYP369B3 resulted in increased mortality in adult females after insecticide exposure. The results confirmed the vital roles of P450s in parasitoid wasps when they were subjected to insecticides. The present study can lay a foundation for an in-depth understanding of the function of P450s in parasitoid wasps.

Supplementary Materials: The following are available online at https:/ /www.mdpi.com/article/10 .3390/insects12070651/s1, Table S1: Primers used for real-time quantitative PCR, Table S2: Primers used for RNA interference.

Author Contributions: Conceived and designed the experiments: S.S. and J.W. Performed the experiments: X.X., M.Y., H.P., and S.S. Analyzed the data: F.W. and S.S. Contributed reagents/materials / analysis tools: J.W. and S.S. Wrote the paper: X.X., M.Y. and S.S. All authors have read and agreed to the published version of the manuscript.

Funding: This study was supported by the Undergraduate Innovation and Entrepreneurship Training Program (201910289010Z).

Conflicts of Interest: The authors declare no conflict of interest. 


\section{References}

1. Feyereisen, R. Insect P450 Enzymes. Annu. Rev. Entomol. 1999, 44, 507-533. [CrossRef]

2. Shaik, S.; Cohen, S.; Wang, Y.; Chen, H.; Kumar, D.; Thiel, W. P450 Enzymes: Their Structure, Reactivity, and Selectivity—Modeled by QM/MM Calculations. Chem. Rev. 2010, 110, 949-1017. [CrossRef]

3. Feyereisen, R. Insect CYP genes and P450 enzymes. Biochem. Mol. Biol. 2011, 8, 236-316.

4. Li, X.; Schuler, M.A.; Berenbaum, M.R. Molecular Mechanisms of Metabolic Resistance to Synthetic and Natural Xenobiotics. Annu. Rev. Entomol. 2007, 52, 231-253. [CrossRef] [PubMed]

5. Bass, C.; Field, L.M. Gene amplification and insecticide resistance. Pest Manag. Sci. 2011, 67, 886-890. [CrossRef] [PubMed]

6. Liu, S.; Zhang, Y.X.; Wang, W.L.; Cao, Y.; Li, S.; Zhang, B.X.; Li, S.G. Identification of putative cytochrome P450 monooxygenase genes from the small white butterfly, Pieris rapae (Lepidoptera: Pieridae), and their response to insecticides. Arch. Insect Biochem. 2018, 98, e21455. [CrossRef] [PubMed]

7. Lu, K.; Cheng, Y.; Li, W.; Li, Y.; Zeng, R.; Song, Y. Activation of CncC pathway by ROS burst regulates cytochrome P450 CYP6AB12 responsible for $\lambda$-cyhalothrin tolerance in Spodoptera litura. J. Hazard. Mater. 2020, 387, 121698. [CrossRef]

8. Wang, Q.; Wang, M.; Jia, Z.; Ahmat, T.; Xie, L.; Jiang, W. Resistance to neonicotinoid insecticides and expression changes of eighteen cytochrome P450 genes in field populations of Bemisia tabaci from Xinjiang, China. Entomol. Res. 2020, 50, 204-211. [CrossRef]

9. Suh, C.P.C.; Orr, D.B.; Van Duyn, J.W. Effect of insecticides on Trichogramma exiguum (Hymenoptera: Trichogrammatidae) preimaginal development and adult survival. J. Econ. Entomol. 2000, 93, 577-583. [CrossRef]

10. Sheng, S.; Feng, S.; Meng, L.; Li, B. Departure mechanisms for host search on high-density patches by the parasitoid, Meteorus pulchricornis. J. Insect Sci. 2014, 14, 205. [CrossRef]

11. Zhang, X.; Zhang, J.; Shao, Y.; Xing, X.; Wang, J.; Liu, Z.; Li, Y.; Ofori, A.D.; Tu, Q.; Wang, J.; et al. Identification of glutathione-Stransferase genes by transcriptome analysis in Meteorus pulchricornis (Hymenoptera: Braconidae) and their expression patterns under stress of phoxim and cypermethrin. Comp. Biochem. Phys. D 2019, 31, 100607. [CrossRef] [PubMed]

12. Liu, Y.; Li, B. Developmental interactions between Spodoptera exigua (Noctuidae: Lepidoptera) and its uniparental endoparasitoid, Meteorus pulchricornis (Braconidae: Hymenoptera). Biol. Control. 2006, 38, 269. [CrossRef]

13. Sheng, S.; Liao, C.W.; Zheng, Y.; Zhou, Y.; Xu, Y.; Song, W.M.; He, P.; Zhang, J.; Wu, F.A. Candidate chemosensory genes identified in the endoparasitoid Meteorus pulchricornis (Hymenoptera: Braconidae) by antennal transcriptome analysis. Comp. Biochem. Phys. D 2017, 22, 20-31. [CrossRef]

14. Shen, J.L.; Wu, Y.D. Pesticide Resistance and Management of Helicoverpa Armigera; China Agricultural Press: Beijing, China, 1995; pp. 91-94.

15. Tamura, K.; Peterson, D.; Peterson, N.; Stecher, G.; Nei, M.; Kumar, S. MEGA5: Molecular evolutionary genetics analysis using Maximum Likelihood, Evolutionary Distance, and Maximum Parsimony Methods. Mol. Biol. Evol. 2011, 28, 2731-2739. [CrossRef]

16. Sheng, S.; Wang, J.; Zhang, X.; Liu, Z.; Yan, M.W.; Shao, Y.; Zhou, J.; Wu, F.A.; Wang, J. Evaluation of sensitivity to phoxim and cypermethrin in an endoparasitoid, Meteorus pulchricornis (Wesmael) (Hymenoptera: Braconidae), and its parasitization Efficiency Under Insecticide Stress. J. Insect Sci. 2021, 21, 1-8. [CrossRef]

17. Qian, X.L. Experimental study on the control effect of $24 \%$ chlorfenapyr suspending agent on the mulberry pest, Glyphodes pyloalis (Walker). China Seric. 2012, 33, 48-49.

18. Livak, K.J.; Schmittgen, T.D. Analysis of relative gene expression data using realtime quantitative PCR and the $2^{-\Delta \Delta C T}$ method. Methods 2001, 25, 402-408. [CrossRef]

19. Team, R.C. R: A Language and Environment for Statistical Computing; R Foundation for Statistical Computing: Vienna, Austria, 2015.

20. Yang, T.; Liu, N. Genome analysis of cytochrome P450s and their expression profiles in insecticide resistant mosquitoes, Culex quinquefasciatus. PLoS ONE 2011, 6, e29418. [CrossRef] [PubMed]

21. Yang, X.; Deng, S.; Wei, X.; Yang, J.; Zhao, Q.; Yin, C.; Du, T.; Guo, Z.; Xia, J.; Yang, Z.; et al. MAPK-directed activation of the whitefly transcription factor CREB leads to P450-mediated imidacloprid resistance. Proc. Natl. Acad. Sci. USA 2020, 117, 10246-10253. [CrossRef]

22. Zhao, Y.; Wang, Z.; Zhu, J.; Liu, N. Identification and characterization of detoxification genes in two cerambycid beetles, Rhaphuma horsfieldi and Xylotrechus quadripes (Coleoptera: Cerambycidae: Clytini). Comp. Biochem. Physiol. Part B 2020, $243,110431$. [CrossRef] [PubMed]

23. Vlogiannitis, S.; Mavridis, K.; Dermauw, W.; Snoeck, S.; Katsavou, E.; Morou, E.; Harizanis, P.; Swevers, L.; Hemingway, J.; Feyereisen, R.; et al. Reduced proinsecticide activation by cytochrome P450 confers coumaphos resistance in the major bee parasite Varroa destructor. Proc. Natl. Acad. Sci. USA 2021, 118, e2020380118. [CrossRef] [PubMed]

24. Vatanparast, M.; Ahmed, S.; Lee, D.; Hwang, S.H.; Hammock, B.; Kim, Y. EpOMEs act as immune suppressors in a lepidopteran insect, Spodoptera exigua. Sci. Rep. 2020, 10, 1-19. [CrossRef]

25. Wu, L.; Yu, Z.; Jia, Q.; Zhang, X.; Ma, E.; Li, S.; Zhu, K.Y.; Feyereisen, R.; Zhang, J. Knockdown of LmCYP303A1 alters cuticular hydrocarbon profiles and increases the susceptibility to desiccation and insecticides in Locusta migratoria. Pestic. Biochem. Phys. 2020, 168, 104637. [CrossRef]

26. Claudianos, C.; Ranson, H.; Johnson, R.M.; Biswas, S.; Schuler, M.A.; Berenbaum, M.R.; Feyereisen, R.; Oakeshott, J.G. A deficit of detoxification enzymes: Pesticide sensitivity and environmental response in the honeybee. Insect Mol. Biol. 2006, 15, 615-636. [CrossRef] [PubMed] 
27. Tarès, S.; Bergé, J.B.; Amichot, M. Cloning and Expression of Cytochrome P450 Genes Belonging to the CYP4 Family and to a Novel Family, CYP48, in Two Hymenopteran Insects, Trichogramma cacœciae and Apis mellifera. Biochem. Biophys. Res. Commun. 2000, 268, 677-682. [CrossRef]

28. Oakeshott, J.G.; Johnson, R.M.; Berenbaum, M.R.; Ranson, H.; Cristino, A.S.; Claudianos, C. Metabolic enzymes associated with xenobiotic and chemosensory responses in Nasonia vitripennis. Insect Mol. Biol. 2010, 19, 147-163. [CrossRef] [PubMed]

29. Yin, C.; Ye, X. Evolution Analysis of Cytochrome P450 Gene Family in Parasitoid Wasps. Chin. J. Biol. Control. 2019, 35, 335-342.

30. Wu, Z.; Pu, X.; Shu, B.; Bin, S.; Lin, J. Transcriptome analysis of putative detoxification genes in the Asian citrus psyllid, Diaphorina citri. Pest Manag. Sci. 2020, 76, 3857-3870. [CrossRef]

31. Kubota, A.; Stegeman, J.J.; Goldstone, J.V.; Nelson, D.R.; Kim, E.Y.; Tanabe, S.; Iwata, H. Cytochrome P450 CYP2 genes in the common cormorant: Evolutionary relationships with 130 diapsid CYP2 clan sequences and chemical effects on their expression. Comp. Biochem. Phys. C 2011, 153, 280-289. [CrossRef]

32. Fang, Z.; Moural, T.W.; Shah, K.; Palli, S.R. Integrated analysis of cytochrome P450 gene superfamily in the red flour beetle, Tribolium castaneum. BMC Genom. 2013, 14, 1-12.

33. Feyereisen, R. Evolution of insect P450. Biochem. Soc. Trans. 2006, 34, 1252. [CrossRef] [PubMed]

34. Shi, T.; Burton, S.; Wang, Y.; Xu, S.; Zhang, W.; Yu, L. Metabolomic analysis of honey bee, Apis mellifera L. response to thiacloprid. Pestic. Biochem. Phys. 2018, 152, 17-23. [CrossRef] [PubMed]

35. Wang, L.J.; Ye, C.L.; Zheng, M.H.; Huang, L.; Zhang, L.F.; Lui, H. Research advances in Drosophila drug resistance mediated by cytochrome P450. Genom. Appl. Biology. 2019, 38, 1304-1309.

36. Hatano, R.; Scott, J.G. Anti-P450lpr Antiserum Inhibits the Activation of Chlorpyrifos to Chlorpyrifos Oxon in House Fly Microsomes. Pestic. Biochem. Phys. 1993, 45, 228-233. [CrossRef]

37. Joußen, N.; Schuphan, I.; Schmidt, B. Metabolism of Methoxychlor by the P450-Monooxygenase CYP6G1 Involved in Insecticide Resistance of Drosophila melanogaster after Expression in Cell Cultures of Nicotiana tabacum. Chem. Biodivers. 2010, 7, 722-735. [CrossRef]

38. Ding, Z.; Wen, Y.; Yang, B.; Zhang, Y.; Liu, S.; Liu, Z.; Han, Z. Biochemical mechanisms of imidacloprid resistance in Nilaparvata lugens: Over-expression of cytochrome P450 CYP6AY1. Insect Biochem. Mol. Biol. 2013, 43, 1021-1027. [CrossRef]

39. Liao, X.; Xu, P.F.; Gong, P.P.; Wan, H.; Li, J.H. The current susceptibilities of brown planthopper Nilaparvata lugens to triflumezopyrim and other frequently used insecticides in China. Insect Sci. 2021, 28, 115-126. [CrossRef]

40. Qiu, X.H. Molecular mechanisms of insecticide resistance mediated by cytochrome P450s in insects. Acta. Entomol. Sin. 2014, $57,477-482$.

41. Liu, J.; Su, X. Effects of chlorantraniliprole and fipronil on cytochrome P450 genes in oriental armyworm Mythimna separata. J. Plant Prot. 2019, 46, 563-573.

42. Sun, H.H.; Yang, B.J.; Zhang, Y.X.; Liu, Z.W. Metabolic resistance in Nilaparvata lugens to etofenprox, a non-ester pyrethroid insecticide. Pestic. Biochem. Phys. 2017, 136, 23-28. [CrossRef]

43. Li, X.X.; Li, R.; Zhu, B.; Gao, X.W.; Liang, P. Overexpression of cytochrome P450 CYP6BG1 may contribute to chlorantraniliprole resistance in Plutella xylostella (L.). Pest Manag. Sci. 2018, 74, 1386-1393. [CrossRef] [PubMed]

44. Mao, K. Metabolic Resistance and Regulation Mechanism of Nitenpyram in Nilaparvata Lugens. Ph.D. Thesis, Huazhong Agricultural University, Wuhan, China, 2020.

45. Liao, X.; Xu, P.; Li, Z.; Ali, E.; Wan, H.; Li, J. Overexpression of CYP6ER1 associated with clothianidin resistance in Nilaparvata lugens (Stal). Pestic. Biochem. Phys. 2019, 154, 39-45. 